\title{
Europa's FUV auroral tail on Jupiter
}

\author{
D. Grodent, ${ }^{1}$ J.-C. Gérard, ${ }^{1}$ J. Gustin, ${ }^{1}$ B. H. Mauk, ${ }^{2}$ J. E. P. Connerney, ${ }^{3}$ and J. T. Clarke ${ }^{4}$ \\ Received 14 December 2005; revised 17 January 2006; accepted 2 February 2006; published 17 March 2006.
}

[1] Ultraviolet images of Jupiter's northern aurora obtained in 2005 confirm the existence of an electromagnetic interaction between Europa and the Jovian ionosphere. The auroral signature shows a two-component structure: a quasi-circular Europa spot, followed by a previously undetected faint tail emission trailing in the direction of corotation flow. The characteristic brightness for the auroral spot is $\sim 14 \pm 1 \mathrm{kR}$ above background, and approximately $7 \pm 1 \mathrm{kR}$ for the tail. The spot's size is $\sim 1100 \mathrm{~km}$, magnetically mapping to an interaction region $\leq 15$ Europa diameters. The auroral tail extends over $\sim 5000 \mathrm{~km}$, which maps along a region of at least 70 Europa diameters. The ultraviolet power emitted by both components varies from a fraction to several GW. The present study suggests auroral interaction at Europa similar to that at Io, but scaled-down by an order of magnitude, including a subcorotating plasma plume in the geometrical wake of Europa. Citation: Grodent, D., J.-C. Gérard, J. Gustin, B. H. Mauk, J. E. P. Connerney, and J. T. Clarke (2006), Europa's FUV auroral tail on Jupiter, Geophys. Res. Lett., 33, L06201, doi:10.1029/2005GL025487.

\section{Introduction}

[2] For more than a decade cameras on the Hubble Space Telescope (HST) have provided images of Jupiter's farultraviolet (FUV) auroral emissions with improving spatial resolution and sensitivity [e.g., Clarke et al., 2004, and references therein]. Auroral emissions are associated with at least three different regions: (1) satellite footprints, (2) the main oval, and (3) emissions located poleward of the main oval. Other sub-structures within these general features are tentatively associated with local phenomena such as dayside and nightside reconnection [Grodent et al., 2003a, 2003b, 2004]. The bright and localized footprint of Io has been extensively described [e.g., Connerney et al., 1993], but the much fainter footprints of Ganymede and Europa have only been identified in a small subset of Space Telescope Imaging Spectrograph (STIS) images [Clarke et al., 1998; Clarke, 2002]. Callisto's footprint has not been detected thus far in FUV images.

[3] Io's volcanism produces copious quantities of neutral gas and plasma (as much as $500-1000 \mathrm{~kg} \mathrm{~s}^{-1}$ [Smyth and Marconi, 2000]). This material escapes Io by means of

\footnotetext{
${ }^{1}$ Laboratoire de Physique Atmosphérique et Planétaire, Université de Liège, Liege, Belgium.

${ }^{2}$ Applied Physics Laboratory, Johns Hopkins University, Laurel, Maryland, USA.

${ }^{3}$ Laboratory for Extraterrestrial Physics, NASA Goddard Space Flight Center, Greenbelt, Maryland, USA.

${ }^{4}$ Center for Space Physics, Boston University, Boston, Massachusetts, USA.

Copyright 2006 by the American Geophysical Union. 0094-8276/06/2005GL025487\$05.00
}

impacts by hot plasma and fast-flowing warm and cold plasma particles and feeds clouds of neutral $\mathrm{O}$ and $\mathrm{S}$ gases that orbit Jupiter in the vicinity of Io, and the Io torus of dense $\mathrm{S}$ and $\mathrm{O}$ plasmas. A fraction of the gases are ionized very close to Io as part of the escape process, resulting in strong mass loading and related plasma effects, which in turn produces strong near-Io perturbations to the local magnetic field. The perturbations caused by this loading, along with the perturbations that occur simply as a result of the motion of a conducting moon though the Io plasma torus, propagate away from Io in the form of Alfvén wings that carry strong field aligned currents to Jupiter's ionosphere. The formation of a localized parallel electric field [Crary, 1997] is thought to accelerate particles to a few tens of $\mathrm{keV}$ [Gérard et al., 2002] and precipitate them in the Jovian ionosphere where they produce an auroral spot-like footprint. In addition to this footprint, a FUV emission tail in the wake of Io's magnetic footprint extends for at least $100^{\circ}$ in longitude along the magnetic footprint of Io's orbit [Clarke, 2002]. This emission tail may be interpreted as the signature of a steady state decoupling process where the subcorotating flux tubes downstream of Io are slowly accelerated up to corotation [e.g., Delamere et al., 2003]. It has also been suggested to arise from wake reacceleration [Hill and Vasyliũnas, 2002], or slow mode waves or shock waves excited by Io's motion through the plasma torus [Erkaev et al., 2002].

[4] A very similar process apparently occurs at Europa. Evidence for plasma and gas releases from Europa includes plasma density and temperature enhancements observed near Europa's orbit during the Voyager epoch [Bagenal, 1992], pickup ions and other local plasma effects observed in close proximity to Europa by Galileo [Paterson et al., 1999; Kurth et al., 2001; Eviatar and Paranicas, 2006], and in situ and remote sensing detection of a gas torus in the vicinity of Europa's orbit of comparable density to that of Io [Mauk et al., 2004]. Quantitative analyses [Schreier et al., 1993; Saur et al., 1999] suggest that the gas/plasma escaping from Europa are of order $50 \mathrm{~kg} \mathrm{~s}^{-1}$, a factor of 10 to 20 less than the gas/plasma escaping from Io. One might therefore anticipate that the strength of the interaction between Europa and its environment is smaller than that of Io by a factor that scales with this gas/plasma release rate.

[5] In this study we report the first detection of Europa's auroral emission tail downstream of Europa's magnetic spot, and suggest it is associated with the corotation enforcement of the slow flux tubes in Europa's wake.

\section{Observations and Data Reduction}

[6] The Advanced Camera for Surveys (ACS) on HST obtained 77 FUV images of Jupiter's northern aurora between 18 April 2005 and 6 May 2005. The images were organized in five HST orbits comprising 15 or 16 contiguous 
Table 1. Timing of the Observations ${ }^{\mathrm{a}}$

\begin{tabular}{ccccc}
\hline Orbit & Date and Time & Images & $\begin{array}{c}\text { CML (S3), } \\
\text { deg }\end{array}$ & $\begin{array}{c}\text { Europa OLG } \\
\text { (S3), deg }\end{array}$ \\
\hline 1 & $18 / 04 / 2005: 1113$ & 15 & 118.6 & 172.1 \\
2 & $18 / 04 / 2005: 1243$ & 16 & 173.3 & 233.0 \\
3 & $25 / 04 / 2005: 1108$ & 15 & 90.2 & 133.8 \\
4 & $25 / 04 / 2005: 1239$ & 16 & 144.9 & 194.8 \\
5 & $06 / 05 / 2005: 0605$ & 15 & 123.6 & 181.7 \\
\hline
\end{tabular}

${ }^{a} U T$ date and time $(\mathrm{dd} / \mathrm{mm} / \mathrm{yyyy}: \mathrm{hhmm})$. All values correspond to the beginning of the first image of the orbit. OLG of Europa is its S3 orbital longitude.

exposures (see Table 1). They were obtained with the photoncounting Multi-Anode Microchannel Array of the High Resolution Camera Solar Blind Channel (SBC). The average plate scale of $\sim 0.032^{\prime \prime}$ per pixel provides a field of view of $35^{\prime \prime} \times 31^{\prime \prime}$ which is comparable to Jupiter's equatorial diameter of $\sim 40^{\prime \prime}$. The transverse distance subtended by one pixel at Jupiter is $\sim 100 \mathrm{~km}$, whereas the resolution element is approximately $0.1 \operatorname{arcsec}(\sim 300 \mathrm{~km})$. All images were taken with the F125LP long-pass filter which is sensitive to the $\mathrm{H}_{2}$ Lyman and Werner bands but rejects the emission shortward of $125 \mathrm{~nm}$, therefore mostly excluding the $\mathrm{H}$ Lyman $-\alpha$ line. All images were obtained with exposure times of $100 \mathrm{~s}$ or $150 \mathrm{~s}$. Following image processing, ACS counts were converted to flux using a factor of $3.47 \times 10^{-3}$ counts per pixel per second for $1 \mathrm{kR}$ of $\mathrm{H}_{2}$. This conversion factor is calculated based on a synthetic UV spectrum of $\mathrm{H}_{2}$ [Gérard et al., 2002] and assumes a square pixel of $0.0301^{\prime \prime}$. Direct comparison of the integrated system throughput for SBC/F125LP and STIS/SrF2 (the equivalent mode on STIS with the SrF2 filter) shows that $\mathrm{SBC}$ is about twice as sensitive as STIS, but with less solar light rejection at the longest wavelengths. This increased sensitivity allows one to detect fainter auroral emission features standing above the sunlight reflected by the Jovian disk.

\section{Detection of Europa's UV Spot and Trailing Tail}

[7] 45 of the 77 ACS images evidence emission associated with the electromagnetic interaction between Europa and Jupiter. As an example, Figure 1 shows the sum of the last three images obtained during orbit 4. Addition of contiguous images increases the signal to noise ratio. In this case, the cumulative exposure time is $410 \mathrm{sec}$. during which the central meridian longitude (CML) at Jupiter increased from $165.8^{\circ}$ to $169.9^{\circ}$. The resulting motion of the auroral region was compensated by shifting the raw images by 9 pixels. The three basic auroral structures described in the introduction (Io footprint and tail, main oval, polar emissions) have been saturated in the display in order to enhance the contrast of the fainter emissions. Two sub-structures appear on the afternoon side between the main oval and the bright footprint of Io: one is likely to be the auroral signature of plasma injection near the orbit of Ganymede [Mauk et al., 2002]; the other, faintest feature forms an isolated spot which we identify as Europa's footprint, followed by a short trailing emission.

[8] The main difficulty in detecting Europa's auroral signature stems from its location close to the equatorial boundary of the main oval, where diffuse and less structured emission masks faint isolated structures. In addition, Europa's footprint appears in a region where auroral signatures produced by transient plasma injections are often observed; these can easily be mistaken for a satellite footprint. To address the first limitation, the observing program was designed to display the footprints of Europa and (primarily) Io between $90^{\circ}$ and $140^{\circ}$ System III (S3), that is in a region largely devoid of emission (as seen in Figure 1) [Clarke et al., 1998; Grodent et al., 2003a] and where the satellite is close to the plasma disk. The second limitation was overcome by repeating the same observing geometry at three different dates to identify the auroral signature of random plasma injections. We used two criteria to decide whether an emission is related to Europa: (1) the observed emission must reasonably match the footprint location predicted by the VIP4 magnetic model [Connerney et al., 1998], and (2) the locus of the different traces must form a continuous line that map magnetically to the same distance in the equatorial plane.

\subsection{Observed Signatures}

[9] A total of 45 positive detections were obtained when the CML was between $120^{\circ}$ and $170^{\circ}$, and the system III longitude of Europa was in the $230^{\circ}-240^{\circ}$ range. They correspond to the 15 images of orbit 1 , the 16 images of orbit 4, and the 14 last images of orbit 5 (see Table 1). Comparison between the location of the observed FUV signatures and the location of Europa's footprint predicted by VIP4 shows that the observed and predicted S3 longitudes match within $\sim 10^{\circ}$, and the observed and predicted Jovicentric latitudes agree to $\sim 2^{\circ}$. The longitude and latitude deviations between predicted and observed positions are the same in orbits 1 and 5, which are the closest in terms of CML though they were obtained 18 days apart. These small and systematic deviations strongly suggest that the observed isolated auroral features, such as that appearing in Figure 1, are indeed mapping to the interaction region between Europa and the magnetospheric plasma.

[10] The images were projected following the method described by Grodent et al. [2003a, 2005]. The accuracy of the limb fitting procedure is limited to $\sim 5^{\circ}$ in longitude and/ or latitude near the limb, but is usually around $\sim 1^{\circ}$ on the disk near the CML. Figure 2 represents the sum of all 45 images, showing the Europa spot and tail projected onto a polar map, fixed in S3 (a reference frame rotating with Jupiter's magnetic field). Figure 2 shows an average auroral morphology that compares well with previous observation

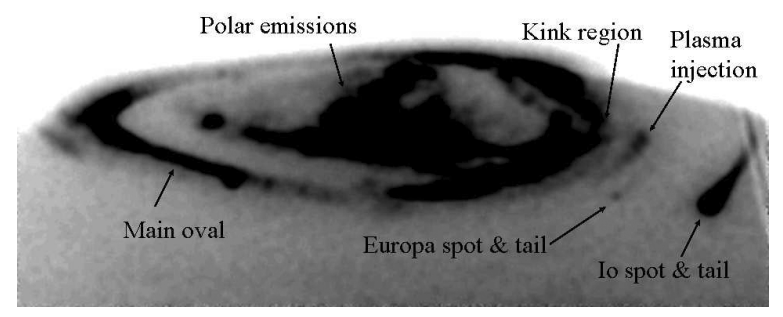

Figure 1. Sum of the last three images obtained during orbit 4 . The Io spot and tail, main oval and polar emissions have been saturated in order to enhance the contrast of the fainter emissions. 


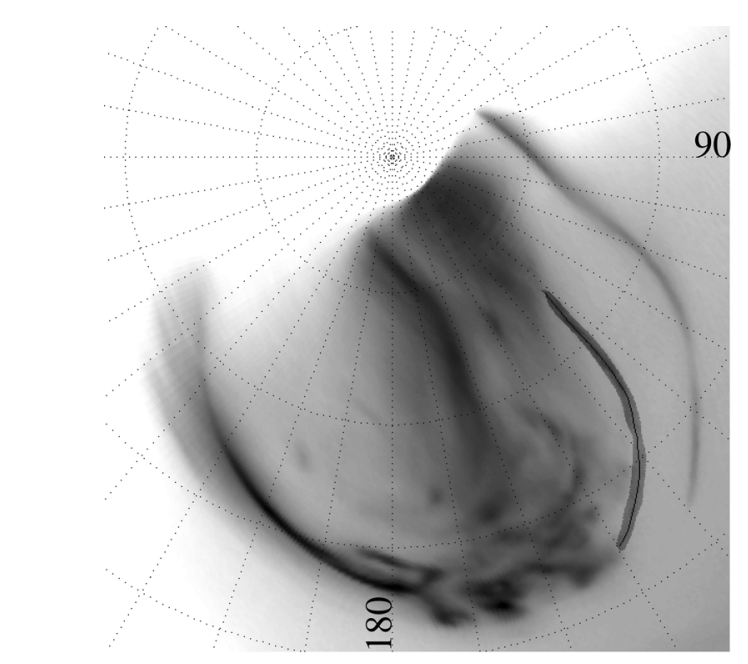

Figure 2. Polar orthographic projection (S3 longitudes) of the sum of the 45 images showing the Europa spot and tail. The footprint of Io and its tail form the long continuous trace appearing at longitudes smaller than $140^{\circ}$. The emission associated with Europa forms a similar trace between the equatorial boundary of the main auroral emission and Io's footprint locus. The central solid line connects the brightest emission points. A grid of $10^{\circ}-$ spaced planetocentric latitudes and longitudes is overlaid, with S3 longitude $180^{\circ}$ toward the bottom and $90^{\circ}$ toward the right.

campaigns [Grodent et al., 2003a]. The footprint of Io and its tail, which appears at different longitudes, contributes to the long continuous trace located at longitudes smaller than $140^{\circ}$. The emission features associated with Europa form a similar trace between the equatorial boundary of the main oval and Io's footprint. The central solid line joins the brightest emission points and is used in the next section to derive lightcurves along the path. The continuity of the path and its striking similarity with Io's strongly argue for the identification of these features (i.e., the spot and the trailing tail) with Europa's auroral signature. The leading and lagging edges of the Europa emission track in Figure 2 merge into the auroral diffuse emission, illustrating the difficulty of detecting Europa's signature outside this sector.

\subsection{Brightness Along Europa's Emission Footpath}

[11] In order to get a higher signal to noise ratio for the Europa spot and tail brightness estimates, we added the signals extracted from the 16 images of orbit 4 . This orbit is most favorable in terms of spot and tail visibility. The average brightness distribution shown in Figure 3 is given in units of $\mathrm{kR}$ of $\mathrm{H}_{2}$ above the local background as a function of the distance along Europa's signature, in $\mathrm{km}$ on the planet. A strip $\sim 2000 \mathrm{~km}$ wide was used to include all emissions from the Europa footprint. The resulting brightness distribution unambiguously shows the presence of the bright spot followed by a fainter tail. The intensity distribution of the spot has Gaussian shape with a peak of $14 \pm 1 \mathrm{kR}$ and a FWHM of approximately $1100 \mathrm{~km}$. The tail extends over $\sim 5000 \mathrm{~km}$ and lies approximately $7 \pm 1 \mathrm{kR}$ above the background level. When mapped to the orbit of Europa with the VIP4 model (assuming magnetic flux conservation), the average spot's size corresponds to $\sim 50000 \mathrm{~km}$, that is $\sim 15$ Europa diameters, and the extent of the emission tail maps to $\sim 200000 \mathrm{~km}$, or $\sim 70$ Europa diameters. It should be noted that the mapped dimension of the spot represents an upper limit since the dimension of the auroral spot derived from the sharpest individual lightcurves is usually smaller than the average. The opposite is true for the emission tail, since it rapidly becomes difficult to discriminate from the background emission. As a result, the brightness within the decreasing phase of the curve is getting smaller than the error bar, which increases with decreasing signal. The emitted power from the spot alone is on the order of $0.8 \mathrm{GW}$. For the tail emission, assuming a rectangular shape of 1000 by $5000 \mathrm{~km}^{2}$, the emitted power is about $0.5 \mathrm{GW}$. The same method applied to the auroral spot of Io provides an average peak brightness of $260 \mathrm{kR}$, in agreement with previous measurements [e.g., Gérard et al., 2006].

\section{Discussion}

[12] The present study confirms the existence of an electromagnetic interaction between Europa and the Jovian ionosphere. Detailed analysis of the auroral signature of this interaction reveals a two-component structure consisting of a quasi-circular Europa spot, already detected in UV STIS images [Clarke et al., 1998; Clarke, 2002], followed by a previously undetected faint emission trailing in the direction of the corotation flow. The resemblance between Europa and Io's auroral signatures suggest that similar interactions occur at both moons. The spot brightness of Europa represents approximately 5\% of Io's spot: one might see the interaction between Europa and the magnetospheric plasma as a replica of the interaction of Io with the torus plasma, scaled down by a factor of 20. Accordingly, the newly-detected tail emission might be associated with fieldaligned currents transferring momentum from Jupiter's ionosphere to a plasma cloud lagging behind corotation in the wake of Europa. The presence of such a plasma cloud or plume has been suggested by different authors. Recently, Eviatar and Paranicas [2006] made use of Galileo observations to argue that Europa (and Callisto) emits an ionospheric plasma plume which extends in the direction of the

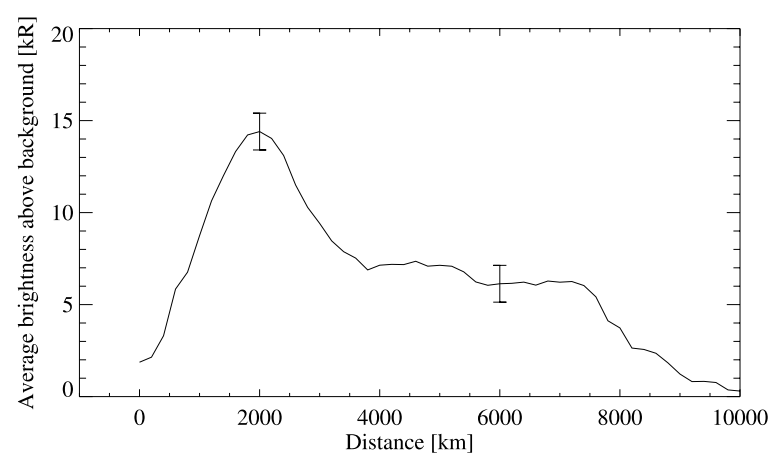

Figure 3. Average brightness distribution obtained from the 16 images of orbit 4 (the most favorable case for spot and tail visibility). The brightness is given in units of $\mathrm{kR}$ of $\mathrm{H}_{2}$ above the local background as a function of the distance along Europa's footpath, in $\mathrm{km}$ on the planet. The error bars were taken as the square root of the local total counts (signal plus background). 
corotating flow. These observations were made when Galileo flew through Europa's geometrical corotation wake, one to two Europa diameters away from the satellite. They further suggest that the plume escaping from Europa is wrapped around Jupiter by corotation and is radially convected by magnetospheric motions. Earlier studies of Europa's wake region have shown the presence of plasma waves typically associated with current-carrying regions [Kurth et al., 2001] as well as evidence of pickup ions [Paterson et al., 1999]. A three-dimensional plasma model describing the interaction of the Jovian magnetosphere with Europa's atmosphere was developed by Saur et al. [1999]. Within the assumptions made in their model, they found that a total electric current of $7 \times 10^{5} \mathrm{~A}$ could be carried by each Alfvén wing, possibly reaching Jupiter's ionosphere and generating Europa's spot. This estimate compares with the current carried at Io of $\sim 5 \times 10^{6} \mathrm{~A}$, in reasonable agreement with the factor of $10-20$ intensity ratio observed between the two spots.

[13] The 45 images showing Europa's signature were obtained when the S3 longitude of Europa was between $65^{\circ}$ and $115^{\circ}$, that is, when Europa was closest to the plasma disk plane. Therefore, the present analysis agrees well with the suggestion made by Kivelson et al. [1999] that Europa's plasma plume is produced only when the moon passes through the plasma disk. This requires a comet-like interaction between Europa and Jupiter's magnetospheric plasma for which the strength of the field-aligned currents coupling Europa to Jupiter's ionosphere and the brightness of a Europa footprint should depend on Europa's position above or below Jupiter's plasma disk.

[14] Acknowledgments. This work is based on observations with the NASA/ESA Hubble Space Telescope, obtained at the Space Telescope Science Institute (STScI), which is operated by AURA, Inc. for NASA under contract NAS5-26555. D.G. and J.C.G. are supported by the Belgian Fund for Scientific Research (FNRS). Partial funding for this research was provided by the PRODEX program of the European Space Agency (ESA). J.T.C. acknowledges funding from NASA under grant HST-GO-10140.01-A from STScI to Boston University. We thank very much both referees for their constructive review of our manuscript.

\section{References}

Bagenal, F. (1992), Giant planet magnetospheres, Annu. Rev. Earth Planet. Sci., 10, 289-328.

Clarke, J. T., et al. (1998), Hubble Space Telescope imaging of Jupiter's UV aurora during the Galileo orbiter mission, J. Geophys. Res., 103, 20,217$20,236$.

Clarke, J. T. (2002), Ultraviolet auroral emissions from the magnetic footprints of Io, Ganymede, and Europa on Jupiter, Nature, 415, 997-1000.

Clarke, J. T., D. Grodent, S. Cowley, E. Bunce, P. Zarka, J. Connerney, and T. Satoh (2004), Jupiter's aurora, in Jupiter: The Planet, Satellites and Magnetosphere, edited by F. Bagenal, B. McKinnon, and T. Dowling, pp. 639-670, Cambridge Univ. Press, New York.

Connerney, J. E. P., R. Baron, T. Satoh, and T. Owen (1993), Images of excited $\mathrm{H}_{3}^{+}$at the foot of the Io flux tube in Jupiter's atmosphere, Science, 262, $1035-1038$.

Connerney, J. E. P., M. H. Acuña, N. F. Ness, and T. Satoh (1998), New models of Jupiter's magnetic field constrained by the Io flux tube footprint, J. Geophys. Res., 103, 11,929-11,939.
Crary, F. J. (1997), On the generation of an electron beam by Io, J. Geophys. Res., 102, 37-49.

Delamere, P. A., F. Bagenal, R. Ergun, and Y.-J. Su (2003), Momentum transfer between the Io plasma wake and Jupiter's ionosphere, J. Geophys. Res., 108(A6), 1241, doi:10.1029/2002JA009530.

Erkaev, N. V., V. S. Semenov, V. A. Shaidurov, D. Langmayr, H. K. Biernat, and H. O. Rucker (2002), Investigation of MHD slow shocks propagating along the Io flux tube, Int. J. Geomagn. Aeron., 3(1), 67-76.

Eviatar, A., and C. Paranicas (2006), The plasma plumes of Europa and Callisto, Icarus, 178, 360-366.

Gérard, J.-C., J. Gustin, D. Grodent, P. Delamere, and J. T. Clarke (2002), Excitation of the FUV Io tail on Jupiter: Characterization of the electron precipitation, J. Geophys. Res., 107(A11), 1394, doi:10.1029/ 2002JA009410.

Gérard, J.-C., A. Saglam, D. Grodent, and J. T. Clarke (2006), The morphology of the ultraviolet Io footprint emission and its control by Io's location, J. Geophys. Res., doi:10.1029/2005JA011327, in press.

Grodent, D., J. T. Clarke, J. Kim, J. H. Waite Jr., and S. W. H. Cowley (2003a), Jupiter's main auroral oval observed with HST-STIS, J. Geophys. Res., 108(A11), 1389, doi:10.1029/2003JA009921.

Grodent, D., J. T. Clarke, J. H. Waite Jr., S. W. H. Cowley, J.-C. Gérard, and J. Kim (2003b), Jupiter's polar auroral emissions, J. Geophys. Res., 108(A10), 1366, doi:10.1029/2003JA010017.

Grodent, D., J.-C. Gérard, J. T. Clarke, G. R. Gladstone, and J. H. Waite Jr. (2004), A possible auroral signature of a magnetotail reconnection process on Jupiter, J. Geophys. Res., 109, A05201, doi:10.1029/ 2003JA010341.

Grodent, D., J.-C. Gérard, S. W. H. Cowley, E. J. Bunce, and J. T. Clarke (2005), Variable morphology of Saturn's southern ultraviolet aurora, J. Geophys. Res., 110, A07215, doi:10.1029/2004JA010983.

Hill, T. W., and V. M. Vasyliũnas (2002), Jovian auroral signature of Io's corotational wake, J. Geophys. Res., 107(A12), 1464, doi:10.1029/ 2002JA009514.

Kivelson, M. G., K. K. Khurana, D. J. Stevenson, L. Bennett, S. Joy, C. T. Russell, R. J. Walker, C. Zimmer, and C. Polanskey (1999), Europa and Callisto: Induced or intrinsic fields in a periodically varying plasma environment, J. Geophys. Res., 104(A3), 4609-4625.

Kurth, W. S., D. A. Gurnett, A. M. Persoon, A. Roux, S. J. Bolton, and C. J. Alexander (2001), The plasma environment of Europa, Planet. Space Sci., 49, 345-363.

Mauk, B. H., J. T. Clarke, D. Grodent, J. H. Waite Jr., C. P. Paranicas, and D. J. Williams (2002), Transient aurora on Jupiter from injections of magnetospheric electrons, Nature, 415, 1003-1005.

Mauk, B. H., D. G. Mitchell, S. M. Krimigis, E. C. Roelof, and C. P. Paranicas (2004), Energetic neutral atoms from a trans-Europa gas torus at Jupiter, Nature, 421, 920-922.

Paterson, W. R., L. A. Frank, and K. L. Ackerson (1999), Galileo plasma observations at Europa: Ion energy spectra and moments, J. Geophys. Res., 104, 22,779-22,791.

Saur, J., F. M. Neubauer, D. F. Strobel, and M. E. Summers (1999), Threedimensional plasma simulation of Io's interaction with the Io plasma torus, J. Geophys. Res., 104, 25,105-25,126.

Schreier, R., A. Eviatar, V. M. Vasyliũnas, and J. D. Richardson (1993), Modeling the Europa plasma torus, J. Geophys. Res., 98, 21,23121,243 .

Smyth, W. H., and M. L. Marconi (2000), Io's oxygen source: Determination from ground-based observations and implications for the plasma torus, J. Geophys. Res., 105, 7783-7792.

J. T. Clarke, Center for Space Physics, Boston University, 725 Commonwealth Avenue, Boston, MA 02215, USA.

J. E. P. Connerney, Laboratory for Extraterrestrial Physics, NASA/GSFC, Greenbelt, MD 20771, USA.

J.-C. Gérard, D. Grodent, and J. Gustin, LPAP, Université de Liège, Allée du 6 Août, 17 (B5c), B-4000- Liège, Belgium. (d.grodent@ulg.ac.be)

B. H. Mauk, Applied Physics Laboratory, Johns Hopkins University, 11100 Johns Hopkins Road, Laurel, MD 20723-6099, USA. 